\title{
Model for the Formation of Helium Bubbles in Palladium
}

\author{
Julio Alfonso Alonso ${ }^{\mathrm{a}, *}$ and Andrés Ayuela ${ }^{\mathrm{b}, \mathrm{c}}$ \\ ${ }^{a}$ Departamento de Física Teórica, Atómica y Optica, Universidad de Valladolid, 47011 Valladolid, Spain \\ ${ }^{\mathrm{b}}$ Centro Mixto CSIC-UPV, 20080 San Sebastián, Spain \\ ${ }^{\mathrm{c}}$ Donostia International Physics Center, 20080 San Sebastián, Spain
}

RECEIVED MAY 24, 2013; REVISED SEPTEMBER 5, 2013; ACCEPTED SEPTEMBER 13, 2013

\begin{abstract}
In implantation experiments or in the inner wall of the vessel of nuclear reactors, irradiation of metals with helium damages the material by forming defects. We focus on studying the formation of $\mathrm{He}$ bubbles by analyzing the energetics of He atoms in bulk palladium and present a simple model for bubble formation. The model emphasizes that the driving force for the formation of $\mathrm{He}$ bubbles is the high energy state of the implanted He.(doi: 10.5562/cca2305)
\end{abstract}

Keywords: helium, palladium, irradiation, bubbles

\section{INTRODUCTION}

The study of helium embedded in metals is important to understand and control the effect of ion irradiation on the metallic parts of the walls of nuclear reactors. Helium may appear in those walls as a result of direct implantation, and also because of the neutron $(n)$ irradiation of the wall materials giving rise to $(n, \alpha)$ reactions emitting $\alpha$ particles, ${ }^{1}$ such as ${ }^{59} \mathrm{Ni}+n \rightarrow{ }^{56} \mathrm{Fe}+\alpha$. In addition, helium may form from tritium by $\beta$ decay. Although helium is insoluble in metals, it can be easily retained in vacancies and voids. Because of its small size, helium atoms can also be trapped at interstitial sites of the metal lattice. If helium is present in sufficient concentrations, vacancies and voids may act as nucleation centers for the formation of liquid He bubbles. Bubbles can grow under suitable conditions of temperature, pressure and defect concentration, ${ }^{2}$ reaching diameters of several nanometers. Formation of bubbles is important in practice, and a high concentration of bubbles affects negatively the mechanical properties of materials. ${ }^{3,4}$ Helium release from the bubbles can occur when the chemical potential of the $\mathrm{He}$ atoms in the bubbles equals that for interstitial $\mathrm{He}$ in the material, and also when the bubbles are large enough to induce the fracture of the matrix. ${ }^{5,6}$ The $\mathrm{He}$ atoms can also cluster at extended defects such as dislocations and grain boundaries.

Among the technologically relevant metals, palladium is interesting because of its resistance to oxidation and to poisoning, as well as for its retention of
${ }^{3} \mathrm{He}^{7}$ The structural changes in Pd alloys due to formation of ${ }^{3} \mathrm{He}$ during tritium storage have been studied, and the local atomic order is only slightly perturbed by the presence of He. Nucleation and growth of He bubbles in Pd have been studied using transmission electron microscopy (TEM) and nuclear magnetic resonance $(\mathrm{NMR}) .{ }^{8}$ Bubbles of $\approx 10-20 \AA$ in diameter have been observed.

Two spectroscopic techniques are mainly used for measuring the $\mathrm{He}$ density in the bubbles, electronenergy-loss spectroscopy (EELS) ${ }^{1,9}$ and vacuum ultraviolet absorption spectroscopy. ${ }^{10}$ Those techniques analyze the energy shift of the absorption line for the $1 s \rightarrow 2 p$ electronic transition of He atoms in the bubbles with respect to the free atoms. ${ }^{11}$ The measured He densities span a wide range of values, between 0.005 and $0.25 \mathrm{He}$ atoms $/ \AA^{3}$, depending on the particular metal host, the conditions of the experiment and the diameter of the bubbles. ${ }^{1,9,12-15}$ In general, high He densities correspond to small bubble diameters, and low He densities to large bubble diameters. For comparison, the density of bulk liquid He measured by Surko et al. ${ }^{16}$ at a temperature of $2 \mathrm{~K}$ and a pressure of $1 \mathrm{bar}$ is 0.02 atoms $/ \AA^{3}$. This means that many bubbles have higher densities than liquid helium. The metallic systems that have been studied are $\mathrm{Al}$ and some transition metals $(\mathrm{Ni}, \mathrm{W})$ and alloys.

In particular, in the case of a Pd-rich alloy $\left(\mathrm{Pd}_{90} \mathrm{Pt}_{10}\right),{ }^{15}$ the He densities vary between 0.015 and $0.035 \mathrm{He}$ atoms $/ \AA^{3}$. For a spherical bubble of radius $R$, the number $n$ of He atoms in the bubble is then between

\footnotetext{
$\dagger$ Dedicated to Professor Douglas Jay Klein on the occasion of his $70^{\text {th }}$ birthday.

* Author to whom correspondence should be addressed. (E-mail: jaalonso@fta.uva.es)
} 
$n=0.015 \times \frac{4}{3} \pi R^{3}$ and $n=0.035 \times \frac{4}{3} \pi R^{3}$ with $R$ in $\AA$. If

the bubble volume corresponds to removing $N$ atoms of the alloy, then the approximate radius of the bubble is $R=R_{\mathrm{ws}} N^{1 / 3}$, where $R_{\mathrm{wS}}=1.52 \AA$ is the Wigner-Seitz cell radius of bulk $\mathrm{Pd}$ (the radius of a sphere with the volume of one atom in the metal). Here we have neglected the difference between Pd and Pt atoms, because their atomic radii are similar. Then, $n$ ranges between $0.22 \mathrm{~N}$ and $0.51 \mathrm{~N}$. Consequently, there are fewer $\mathrm{He}$ atoms in the bubble than the number of Pd atoms displaced to form the void. However, the situation is different in materials in which the He density in the bubbles is high. In those cases, values $n \approx 2 N$ are common.

Atomistic simulations of the formation of bubbles have been performed, ${ }^{17,18}$ but the process of bubble formation is still under investigation. ${ }^{19} \mathrm{We}$ present a simple model for the formation of He bubbles in metals, which we apply specifically to Pd. The model is set up in terms of some properties of the metal, like the vacancy formation energy and others. Values for some of those quantities exist in the literature, either from measurements or from calculations. The values of other quantities necessary to apply the model are not available, and for those we have performed density functional calculations.

\section{DEFECTS PRODUCED BY ION IMPLANTATION}

In high energy $\mathrm{He}^{+}$implantation in metals, a large part of the energy of the impinging ion is lost to electronic processes in the metal. In addition, the moving He ions induce damage in the metal, in particular vacancies and self-interstitials, by collisions mainly near the end of the traveling path of the ions. The helium ion ends up as a neutral interstitial atom somewhat further in the track than the last vacancy it produces.

Defects in pure palladium have been studied before. The measured and estimated vacancy formation energies $E_{v}$ in bulk Pd are in the range $1.4-1.8 \mathrm{eV}^{20-22}$ Theoretical calculations ${ }^{23,24}$ using the density functional formalism $(\mathrm{DFT})^{25}$ give $E_{v}=1.30-1.51 \mathrm{eV}$, and a semiempirical model of Miedema ${ }^{26}$ gives $E_{v}=1.22-$ $1.34 \mathrm{eV}$. The energy of formation of a self-interstitial defect in a Pd crystal is larger. Using the embeddedatom method, Foiles and coworkers ${ }^{22}$ have obtained $E_{\text {self }}=3.42 \mathrm{eV}$. This high value indicates that Pd selfinterstitial defects are rather unstable. Self-interstitials in solid materials are highly mobile; their diffusivities are orders of magnitude higher than the diffusivities of vacancies. $^{27}$ Consequently, a part of the Pd selfinterstitials created during $\mathrm{He}^{+}$implantation will tend to disappear in the process of relaxation of the collision cascade, either by recombination with vacancies or by occupation of new lattice sites at grain boundaries. It is safe to assume that a certain amount of vacancies survives after relaxation of the damage produced by the collision cascade, and that this concentration is larger than the concentration required by statistical mechanics for a crystal in equilibrium. ${ }^{28}$ We expect that the concentration of $\mathrm{Pd}$ self-interstitials will be smaller in comparison.

At the end of their travel through the metal, the implanted He atoms have lost most of their kinetic energy, and those atoms end up, most often, occupying interstitial positions in the crystalline lattice of $\mathrm{Pd}$. The implantation process then leads to a Pd crystal containing a certain number of vacancies, and also He atoms occupying interstitial positions. The state of the $\mathrm{He}$ atom occupying an interstitial position is rather unstable. We have performed density functional calculations of the energy required to insert a He atom in an octahedral interstice. The calculations have been performed with the VASP code, ${ }^{29}$ with a basis of plane waves to expand the electronic wave functions. We have used the generalized gradient approximation (GGA) of PerdewBurke-Ernzerhof $(\mathrm{PBE})^{30}$ for electronic exchange and correlation, and the core electrons have been treated by the projector augmented wave (PAW) method. ${ }^{31}$ An energy cutoff of $300 \mathrm{eV}$ was adopted, which achieves a total energy convergence within several meV. For geometry optimizations, the convergence criterion on the forces was $1.4 \times 10^{-5} \mathrm{eV} / \AA$. The supercell has dimensions $\mathrm{a}=\mathrm{b}=\mathrm{c}=7.78 \AA$; this supercell has 32 atoms in bulk Pd, and 33 in the doped case. A $13 \times 13 \times 13$ Monkhorst-Pack grid is used to sample the Brillouin zone. After introducing the $\mathrm{He}$ interstitial, the dimensions of the supercell are kept unmodified, as usual, ${ }^{32}$ but the positions of all the atoms in the cell are fully relaxed. The formation energy of the He interstitial, that is, the energy neccesary to bring a free $\mathrm{He}$ atom to an octahedral interstice in the metal is $E_{\mathrm{i}}^{\mathrm{He}}=4.22 \mathrm{eV}$. Other recent DFT calculations ${ }^{32,33}$ with a similar number of atoms in the supercell predict values $E_{\mathrm{i}}^{\mathrm{He}}=3.7 \mathrm{eV}^{33}$ and $E_{\mathrm{i}}^{\mathrm{He}}=3.65 \mathrm{eV} .{ }^{32}$ In our opinion, the difference may be due to the broadening used for the density of electronic states, which affects the calculation of the forces on the atoms. In fact, with a looser broadening we find $E_{\mathrm{i}}^{\mathrm{He}}=3.58 \mathrm{eV}$. The value we use through this paper is $E_{\mathrm{i}}^{\mathrm{He}}=4.22 \mathrm{eV}$.

The recombination of a He interstitial with a vacancy

$$
\mathrm{He}_{\text {interstitial }}+\mathrm{Pd}_{\text {vacancy }} \rightarrow \mathrm{He}_{\text {substitutional }} \text {. }
$$

is a favorable process. Using DFT, we have calculated a recombination energy $\Delta E_{\mathrm{r}}=-2.97 \mathrm{eV}$. That is, the recombination releases an energy of nearly $3 \mathrm{eV}$. The reason can be simply understood from the embedded atom model. ${ }^{34,35}$ Helium is an inert gas atom with a $1 \mathrm{~s}^{2}$ 
closed-shell electronic configuration. Embedding this atom in a solid medium costs energy, and that energy increases linearly with the effective electronic density of the medium in which He is embedded. ${ }^{34}$ The electronic density is large at interstitial sites of the Pd crystal, and much smaller at vacancies in the same crystal. This energy of $-2.97 \mathrm{eV}$ represents a strong driving force for the recombination, and consequently there is a strong tendency to fill vacancies with He atoms. The presence of He bubbles in spherical voids in the crystal has the same physical origin.

\section{VACANCIES AND VOIDS}

Let us first consider the formation of voids by the agglomeration of vacancies. ${ }^{36}$ In the theory developed by Miedema, ${ }^{26}$ the formation energy of a vacancy can be viewed as the energy required to form an inner surface in the metal with the dimensions of the surface of an atomic cell, $4 \pi R_{W}^{2}$. That is,

$$
E_{v}=\left(4 \pi R_{W}^{2} \gamma\right) Q
$$

where $\gamma$ is the surface energy. The constant $Q$ is an empirical correction factor that serves to take into account several effects. First of all, the difference between the flat shape of the surface of a metal and the shape of the boundary of a vacancy in the same metal. $Q$ also incorporates the effect of the atomic and electronic relaxation around the vacancy. Both the surface energy and the vacancy formation energy $E_{v}$ can be viewed as the positive energy contribution arising from the electron density mismatch that appears at a surface or at a vacancy. In a macroscopic surface, the electron density changes from the value $\rho_{\text {int }}$ typical of the interstitial region of the metal outside the cores of the atoms to the zero value at the vacuum. On the other hand, in a vacancy, due to its small size, the density does not fall to zero and it retains a finite value at the center of the vacancy. In addition, there is a small relaxation of the neighbor atoms towards the centre of the vacancy, which reduces the effective surface area of the cavity. For all these reasons, the value of $Q$ is different from 1 . We have determined an optimized value of $Q$ for $\mathrm{Pd}$ by using in Eq. (2) the experimental surface energy of 2.1 $\mathrm{J} / \mathrm{m}^{2}$ given by Miedema ${ }^{37}$ and a vacancy formation energy of $1.6 \mathrm{eV}$, which is an average of the measured vacancy formation energies. ${ }^{20-22}$ The resulting value is $Q=0.42$.

The energy to form $N$ independent vacancies is then $N E_{v}=4 \pi R_{W}^{2} \gamma Q N$. On the other hand, the radius $R$ of a spherical void with a volume equal to the sum of the volumes of the $N$ vacancies is $R=R_{\mathrm{W}} N^{1 / 3}$, and the energy necessary to create such a void is

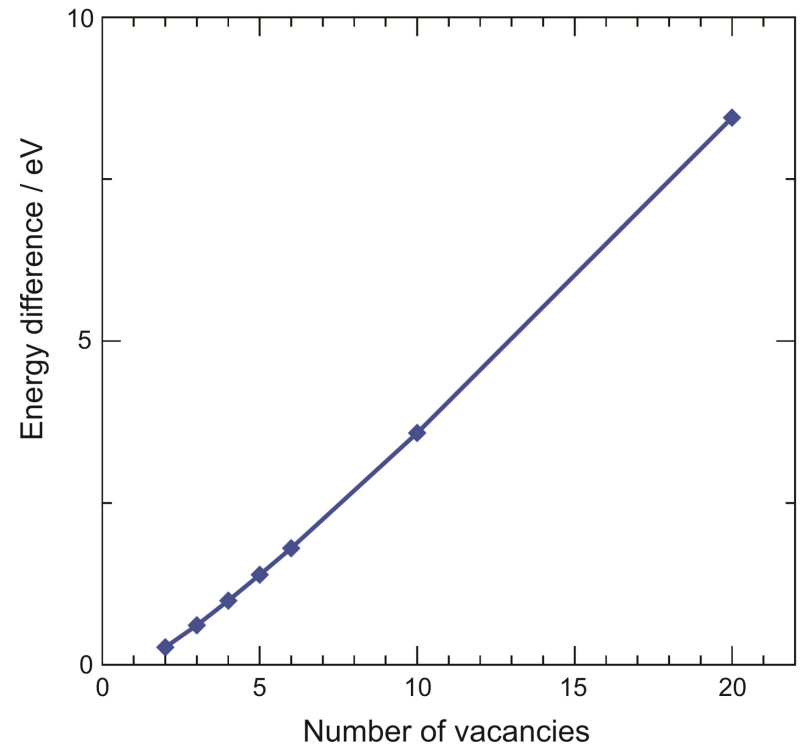

Figure 1. Difference $N E_{v}-E_{\text {hole }}$ between the energy of formation of $N$ isolated vacancies and the energy of formation of a void formed by the agglomeration of those $N$ vacancies, versus $N$.

$$
E_{\text {hole }}=4 \pi R^{2} \gamma Q=4 \pi R_{W}^{2} N^{2 / 3} \gamma Q
$$

One can notice, using Eqs. (2) and (3), that $E_{\text {hole }}=$ $N^{2 / 3} E_{v}$. Consequently, $E_{\text {hole }}<N E_{v}$, the reason being that the amount of surface area newly created is smaller in the case of the void compared to the case of $N$ separated vacancies. Forming the difference $N E_{v}-E_{\text {hole }}$, and using the above value of $Q=0.42$ for $\mathrm{Pd}$ we find

$$
N E_{v}-E_{\text {hole }}=0.67\left(N-N^{2 / 3}\right) .
$$

In Figure 1 we have plotted $N E_{\mathrm{v}}-E_{\text {hole }}$ versus $N$. The Figure shows the substantial energetic stabilization that occurs when $N$ vacancies form a void. A strong driving force then exists for the coalescence of neighbor vacancies to form divacancies (two vacancies in nearest neighbor sites) and then larger voids. There are, however, two effects opposing the formation of a void from vacancies. The first one is the entropy arising from the random distribution of vacancies, which contributes to the free energy of the crystal. However, since vacancies are created during the implantation process, the Pd crystal is expected to be supersaturated; that is, the number of vacancies present is substantially above the equilibrium number at room temperature. The second is the existence of an energy barrier for the diffusion of vacancies; that is, for the hopping of a vacancy from a lattice site to a neighbor site. The vacancy migration energy in $\mathrm{Pd}$, calculated by Foiles and coworkers, ${ }^{22}$ is $E_{\mathrm{m}}(1 v)=0.82 \mathrm{eV}$, and the migration energy of a divacancy is substantially smaller, $E_{\mathrm{m}}(2 v)=0.48 \mathrm{eV}$. 
Under the typical conditions of the implantation experiments, motion of those defects is expected in the structural relaxation step.

An approximation has been used, for simplicity, in Eq. (3). $Q$ has been taken as a constant, but in a strict sense $Q$ depends on the void radius $R$. In fact, $Q$ should be equal to 1 in a macroscopic hole. We have explored this issue by assuming that the value of $Q$ varies linearly between $Q=0.42$ for $N=1$ (the vacancy) and $Q=1$ for a large void with a volume equivalent to the added volume of 1000 vacancies. In this way, we obtain

$$
Q(N)=0.419+0.000581 N
$$

and repeating the calculation of $N E_{v}-E_{\text {hole, a corrected }}$ expression results

$$
\begin{aligned}
& N E_{v}-E_{\text {hole }}= \\
& 0.67\left(N-N^{2 / 3}\right)-0.0016\left(0.58 N^{5 / 3}-N^{2 / 3}\right) .
\end{aligned}
$$

The first term is the same as in Eq. (4), and the second is the correction. This correction lowers the energy difference $N E_{v}-E_{\text {hole }}$ very slightly. For the range of values of $N$ shown in Figure 1, the correction is smaller than 2 percent, and reaches 15 per cent for the largest hole, of $N=1000$. Consequently, the conclusions reached with the Eq. (4) are not modified.

\section{FORMATION OF HELIUM BUBBLES}

Due to the repulsive interaction between the He and the $\mathrm{Pd}$ atoms, the implanted $\mathrm{He}$ atoms located in interstitial positions tend to segregate to the surface of the metal, where the He atoms could evaporate. However, migration to inner defects like vacancies and voids may be much easier. The activation energy barrier for diffusion of He through the palladium lattice is small. In solid metals, the electron density is pretty uniform in the region outside the cores. In fact, the concept of an average electron density in the interstitial regions of metals has been very useful, and its validity rests on the fact that the local deviations from an average electron density are small. ${ }^{35}$ This means that the potential energy surface in the path of a He atom moving from an insterstitial position to another interstitial position is relatively flat and the He atom will not face substantial barriers in its motion. DFT calculations report barriers of only 0.13 or $0.14 \mathrm{eV}^{32}$ As indicated above, the recombination of a He interstitial and a vacancy releases an energy $-\Delta E_{\mathrm{r}}=2.97 \mathrm{eV}$. On the other hand, we have calculated the energy difference between the state of a $\mathrm{He}$ atom trapped in a Pd vacancy and the same atom after migrating to the surface and evaporating to the gas. That energy difference equals $1.25 \mathrm{eV}$, favorable, of course, to the free He. The electron density of $\mathrm{Pd}$ in most of the volume of the void is very small, much smaller than in a single vacancy, and thus the above energy of $1.25 \mathrm{eV}$ can be taken also as an estimate for the energy difference between the He atom trapped in a vacancy and the same atom trapped in a void. The sum $2.97+1.25=4.22 \mathrm{eV}$ is then the energy released when a He interstitial migrates to a void. Consequently, a strong driving force exists for the accumulation of $\mathrm{He}$ atoms in voids.

In addition, another mechanism also induces the growth of a void, with an associated increase of the size of the He bubble inside. This is again due to the energy released when He interstitials become trapped in a void. That trapping energy was estimated above as $4.22 \mathrm{eV}$. We now consider the process of displacing one $\mathrm{Pd}$ atom which forms part of the surface of the void to a nearby interstitial position, producing a vacancy-(Pd self-interstitial) pair which enlarges the volume of the void by a small amount (the volume of the created vacancy). If we estimate the energy to create the vacancy-(Pd self-interstitial) pair defect by just adding $E_{v}$ and $E_{\text {self }}$, this gives an approximate value $E_{v+\text { self }}=E_{v}+E_{\text {self }}=4.7 \mathrm{eV}$. The energy released by trapping a $\mathrm{He}$ atom in the void is not enough to create a vacancy-(Pd self-interstitial) pair. However, trapping two $\mathrm{He}$ atoms is enough to overcome the energy required to form the pair defect. This means that the He atoms can act as a catalyst for the void growth by pushing $\mathrm{Pd}$ atoms away from their lattice positions in the void surface to interstitial sites nearby. The displacement of $\mathrm{Pd}$ atoms then creates vacancies which increase the size of the void. This mechanism of void growth, catalyzed by He, may be even more important than the other mechanism discussed above, the capture of vacancies, because $\mathrm{He}$ atoms move in $\mathrm{Pd}$ much easier than vacancies.

\section{CONCLUSIONS}

In summary, the agglomeration of vacancies to form voids is energetically favorable due to a surface energy effect: the inner surface area of the void is smaller than the sum of the inner surfaces of the separated independent vacancies. Due to the strongly repulsive interaction of the interstitial He atoms in the Pd metal, the accumulation of He atoms in voids is strongly favored. In addition, the concerted trapping of two or more $\mathrm{He}$ atoms catalyzes the growth of the void by displacing Pd atoms.

Acknowledgements. Work supported by MICINN of Spain and the European Regional Development Fund (Grants MAT201122781 and FIS2010-19609-C02-02), Junta de Castilla y Leon (Grant VA158A11-2), Departamento Vasco de Educación and UPV/EHU (Grant IT-366-07), and Departamento Vasco de 
Industria and Diputación Foral de Guipuzcoa under the ETORTEK research program (NANO-IKER Grant IE11-304). We thank P. M. Echenique for helpful comments.

\section{REFERENCES}

1. C. A. Walsh, J. Yuan, and L. M. Brown, Phil. Mag. A 80 (2000) 1507.

2. P. J. Goodhew, Radiat. Effects 78 (1983) 381.

3. A. A. Lucas, Physica B 127 (1984) 225.

4. J. A. Knapp, D. M. Follstaedt, and S. M. Myers, J. Appl. Phys. 103 (2008) 013518.

5. W. G. Wolfer, Phil. Mag. A 58, (1989) 285; 59 (1989) 87.

6. R. C. Bowman and A. Attalla, Phys. Rev. B 16 (1977) 1828.

7. J. A. Emig, R. C. Gaza, L. D. Christensen, P. R. Coronado, and P. C. Souers, J. Nucl. Mater. 187 (1992) 209.

8. C. C. Abell and A. Attalia, Phys. Rev. Lett. 59 (1987) 995.

9. S. Frechard, M. Walls, M. Kociak, J. P. Chevalier, J. Henry, and D. Gorse, J. Nucl. Mater. 393 (2009) 102.

10. S. E. Donnelly, J. C. Rife, J. M. Gilles, and A. A. Lucas, J. Nucl. Mater. 93-94 (1980) 767.

11. N. C. Pyper, D. W. Essex, and C. T. Whelan, Phil. Mag. B 81 (2001) 91

12. W. Jäger, R. Manzke, H. Trinkaus, J. Fink, and G. Crecelius, Radiat. Effects 78 (1983) 315.

13. W. Jäger, R. Manzke, H. Trinkaus, G. Crecelius, and R. Zeller, J. Nucl. Mater. 111-112 (1982) 674.

14. T. Schober, R. Lasser, J. Golczewski, C. Dieker, and H. Trinkaus, Phys. Rev. B 31 (1985) 7109.

15. D. Taverna, M. Kociak, O. Stephan, A. Fabre, E. Finot, B. Decamps, and C. Colliex, Phys. Rev. Lett. 100 (2008) 035301.

16. C. M. Surko, G. J. Dick, F. Rief, and W. C. Walker, Phys. Rev. Lett. 23 (1969) 842.
17. W. D. Wilson, M. I. Baskes, and C. L. Bisson, Phys. Rev. B 13 (1976) 2470

18. W. D. Wilson, C. L. Bisson, and M. I. Baskes, Phys. Rev. B 24 (1981) 2470

19. L. Wang, W. Hua, S. Xiao, J. Yang, and H. Deng, Nucl. Instr. Meth. Phys. Res. B 267 (2009) 3185.

20. T. R. Mattsson and A. E. Mattsson, Phys. Rev B 66 (2002) 214110.

21. H. E. Schaefer, Phys. Status Solidi A 102 (1987) 47.

22. S. M. Foiles, M. I. Baskes, and M. S. Daw, Phys. Rev. B 33 (1988) 7983.

23. R. P. Kauffman and A. M. Rappe, Phys. Rev. B 67 (2003) 085403.

24. X. Zhang and G. Lu, Phys. Rev. B 77 (2008) 174102.

25. W. Kohn and L. J. Sham, Phys. Rev. 130 (1965) A1133.

26. A. R. Miedema, Z. Metallkde 70 (1979) 345.

27. L. M. Brown, Scripta Metall. 6 (1972) 387.

28. L. A. Girifalco, Statistical Mechanics of Solids, Oxford Univ. Press, New York, 2000.

29. G. Kresse and J. Hafner, Phys. Rev. B 48 (1993) 13115; G. Kresse and J. Hafner, Phys. Rev. B 49 (1994) 14251; G. Kresse and J. Furthmüller, Comput. Mater. Sci. 6 (1996) 15.

30. J. P. Perdew, K. Burke, and M. Ernzerhof, Phys. Rev. Lett. 77 (1996) 3865.

31. P. E. Blochl, Phys. Rev. B 50 (1994) 17953.

32. P. Lin, First principles calculations of helium cluster formation in palladium tritides, $\mathrm{PhD}$ Thesis, Georgia Institute of Technology, 2010.

33. X. Zeng, H. Deng, and W. Hu, Nucl. Instr. Meth. Phys. Res. B 267 (2009) 3037.

34. M. J. Stott and E. Zaremba, Phys. Rev. B 22 (1980) 1564.

35. J. A. Alonso and N. H. March, Electrons in Metals and Alloys, Academic Press, London, 1989.

36. M. R. Mruzik and K. C. Russell, Surf. Sci. 67 (1977) 205.

37. A. R. Miedema, Z. Metallkde 69 (1978) 287. 